\title{
Photoacoustic imaging of prostate cancer
}

\author{
Xuanjin Yang \\ Stephenson Research and Technology Center \\ University of Oklahoma Norman, Oklahoma 73019, USA \\ xuanjin.yang-1@ou.edu \\ Liangzhong Xiang* \\ School of Electrical and Computer Engineering \\ University of Oklahoma Norman \\ Oklahoma 73019, USA \\ xianglzh@ou.edu
}

Received 3 June 2017

Accepted 4 June 2017

Published 5 July 2017

\begin{abstract}
Photoacoustic imaging (PAI), also known as optoacoustic imaging, is a rapidly growing imaging modality with potential in medical diagnosis and therapy monitoring. This paper focuses on the techniques of prostate PAI and its potential applications in prostate cancer detection. Transurethral light delivery combined with transrectal ultrasound detection overcomes light scattering in the surrounding tissue and provides optimal photoacoustic signals while minimizing invasiveness. While label-free PAI based on endogenous contrast has promising potential for prostate cancer detection, exogenous contrast agents can further enhance the sensitivity and specificity of prostate cancer PAI. Further in vivo studies are required in order to achieve the translation of prostate PAI to clinical implementation. The minimal invasiveness, relatively low cost, high specificity and sensitivity, and real-time imaging capability are valuable advantages of PAI that may improve the current prostate cancer management in clinic.
\end{abstract}

Keywords: Photoacoustic/optoacoustic imaging; prostate cancer; cancer therapy monitoring; prostate endoscopy; cellular imaging.

\section{Background}

Prostate cancer is the second most common cancer in men as well as the second highest death-causing cancer in men in the United States. ${ }^{1}$ An estimate of
$12.9 \%$ of males in the United States will be diagnosed with prostate cancer throughout their lifespan. ${ }^{1}$ At early stages, prostate cancer may show little or no symptoms, making prostate cancer prognosis and early diagnosis especially important..$^{2-5}$ Prostate

${ }^{*}$ Corresponding author.

This is an Open Access article published by World Scientific Publishing Company. It is distributed under the terms of the Creative Commons Attribution 4.0 (CC-BY) License. Further distribution of this work is permitted, provided the original work is properly cited. 
cancer, however, is difficult to detect or locate due to the obstructed anatomic position of the prostate, the weak imaging contrast of some prostate tumors, and the similarity between malignant tissue and benign prostatic hyperplasia (BPH). Current major screening and diagnostic methods include the prostatespecific antigen (PSA) blood test, ${ }^{6}$ digital rectal examination (DRE), ${ }^{7}$ and transrectal ultrasonography (TRUS) guided biopsy. ${ }^{7}$ PSA blood test examines the level of PSA in blood serum, which is generally elevated in men with prostate cancer. ${ }^{6}$ However, a portion of prostate cancer patients do not have elevated PSA levels, leading to a relatively low sensitivity of the PSA blood test. ${ }^{8,9}$ Serum PSA level is also associated with many factors other than cancer, thus often resulting in false positives. ${ }^{6,10}$ Therefore, PSA blood test must be generally combined with other methods, such as DRE. ${ }^{7,11-14}$ During DRE, the doctor manually examines the size, shape, and texture of the prostate through the rectum. ${ }^{15}$ Morphological abnormalities, such as enlargement and lumps, are often indicative of prostate cancer or BPH. ${ }^{16,17}$ If abnormalities of the prostate or high PSA level are detected, further examination, such as TRUS and TRUS-guided prostate biopsy, may be required for diagnosis. . $^{8}, 11,18$ TRUS is a commonly used method for clinical prostate imaging and provides satisfactory images of the structure of the entire prostate. ${ }^{19}$ Nevertheless, due to the limited contrast of ultrasound (US) imaging, at least $30-40 \%$ of prostate cancer remains invisible under TRUS. ${ }^{19-22}$ Furthermore, TRUS is incapable of differentiating malignant tumors and BPH. ${ }^{19}$ A more important role of TRUS is to visualize the prostate and the biopsy needle during a prostate biopsy, a more invasive procedure in which a sample from the prostate is collected through biopsy needles and then analyzed via pathology. ${ }^{23-26}$ TRUS-guided prostate biopsy is the current gold standard for clinical prostate cancer diagnosis ${ }^{27}$; however, this procedure is not a targeted biopsy and repeated biopsies are often required. ${ }^{25,28-30}$

Magnetic resonance imaging (MRI) has a higher sensitivity than TRUS in prostate cancer detection and may be suitable for targeted imaging-guided prostate biopsies; however, MRI is relatively expensive and time-consuming. ${ }^{31-33}$ Positron emission tomography (PET) is also capable of prostate cancer detection, although PET requires the injection of radiotracers, and the low spatial resolution of PET must be compensated by X-ray computed tomography $(\mathrm{CT}) \cdot{ }^{34,35}$ Due to the difficulty for PET radiotracers to target prostate cancer, $\mathrm{PET} / \mathrm{CT}$ also has limited sensitivity for prostate cancer detection. ${ }^{36}$

In this review, we introduce an emerging imaging modality, photoacoustic imaging (PAI), as a potential tool for the detection of prostate cancer. The review includes: (a) principals of PAI; (b) PAI systems for prostate imaging; (c) PAI of prostate cancer using endogenous contrast; (d) PAI of prostate cancer using exogenous contrast agents, and (e) future outlook.

\section{Principles of PAI}

For the past two decades, PAI has been rapidly developing under focused research by a number of researchers. PAI, also known as optoacoustic imaging, is an innovative, noninvasive hybrid imaging system that holds potential in various fields including, but not limited to, medical diagnosis, therapy monitoring, biological studies, and inorganic imaging. ${ }^{37-47}$ The PAI system is based on the photoacoustic (PA) effect, discovered by Bell in 1880 when he observed sound waves produced by a solid sample exposed to sunlight. ${ }^{48}$ The PA effect is the phenomenon that light induces the generation of US waves in the irradiated object. Generally, in a PAI system, pulsed electromagnetic waves, such as near-infrared (NIR) radiation, induce instantaneous heating in the target. The local heating causes an elastic expansion that generates pressure waves, which are detected by sensitive US transducers. The US signals are then reconstructed into two-dimensional (2D) or three-dimensional (3D) images. ${ }^{49}$

Conventional optical imaging modalities have excellent contrast, yet extensive optical scattering causes substantial loss in resolution as the penetration depth increases. ${ }^{50}$ Pure US imaging benefits from low acoustic scattering, thus having higher penetration depth and spatial resolution. ${ }^{51}$ However, due to the similarity in acoustic properties of biological tissues, pure US imaging generally lacks effective contrast in soft tissue imaging. ${ }^{52}$

In a PAI system, the optical irradiation provides effective contrast among different types of biological tissues, and the collection of acoustic signals instead of optical signals overcomes the weakness of optical signal scattering, providing excellent spatial resolution. As a hybrid imaging system, PAI combines the contrast capability of optical imaging with the 
spatial resolution of US imaging, overcoming the weaknesses of both the conventional imaging modalities. ${ }^{53,54}$ In addition, PAI is capable of nonionizing, label-free, and real-time imaging at a relatively low cost, which are valuable advantages over X-ray imaging, PET, and MRI. It must be noted, however, that the penetration depth of soft tissue PAI is limited by the optical scattering of the light source in biological tissues, and the imaging resolution depends largely on the frequency of the US transducer. ${ }^{54}$

\section{PAI Systems for Prostate Imaging}

Due to optical scattering in the prostate and its surrounding tissue, the penetration depth of prostate PAI is typically limited to $3-5 \mathrm{~cm} .{ }^{55}$ The human prostate is located beyond this penetration limit from the skin surface ${ }^{56}$; therefore, human prostate PAI with an external light source is currently unfeasible. Fortunately, the feasibility of prostate PAI using endoscopic light delivery has been well demonstrated through phantom studies, ${ }^{57,58}$ simulation studies, ${ }^{55,59,60}$ and animal studies. ${ }^{57,58}$ One of the major challenges of human prostate PAI is the delivery of the light source. Ideally, the light source must be capable of illuminating the whole prostate while minimizing invasiveness. To date, the two least invasive light delivery methods are transrectal optical fibers, which can be readily coupled with transrectal US probes ${ }^{64,65}$ and transurethral optical fibers, which can be delivered through urinary catheters. ${ }^{61}$ Utilizing endoscopic light delivery, PAI shows promising potential for in vivo whole-prostate imaging.

Possible configurations of prostate PAI systems include transrectal light delivery and US detection ${ }^{64,65}$

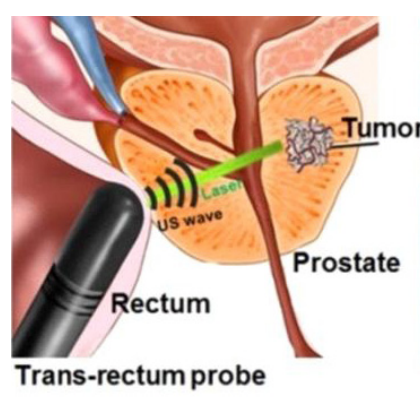

(a) (b)

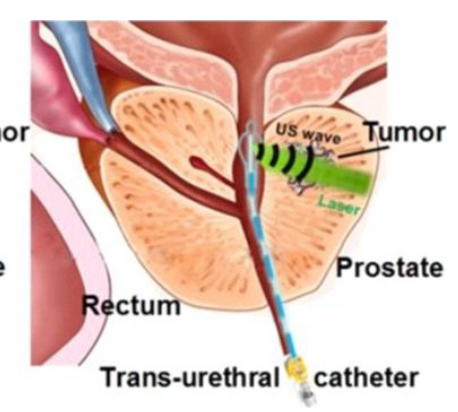

Fig. 1. Configurations of transrectal (a) and transurethral (b) PAI of the prostate. (a) A transrectal US transducer coupled with transrectal light delivery; (b) A transurethral US transducer coupled with transurethral light delivery.
(Fig. 1(a)), transurethral light delivery and US detection $^{55,60}$ (Fig. 1(b)), as well as transurethral light delivery coupled with transrectal US detection. ${ }^{61} \mathrm{Al}-$ though transurethral US detection yielded optimal image quality in Monte Carlo simulations performed by Tang et al. ${ }^{60}$ (Figs. 2(c) and 2(g)) and El-Gohary et al. ${ }^{55}$ a transrectal US transducer is more frequently used in urology. Urologists prefer a transrectal transducer because it is convenient for clinical practice. The feasibility of transrectal US detection has been thus well demonstrated in simulation studies and in vivo. ${ }^{55,57-62,64,65}$

\subsection{Transrectal light delivery}

A transrectal US probe coupled with a transrectal optical fiber is the most convenient and least invasive configuration for human prostate PAI, since the transrectal US probe is already widely used in the clinic. Small-scale clinical trials conducted by Horiguchi et al. ${ }^{64}$ and Ishihara et al. ${ }^{65}$ utilizing a transrectal PAI probe, have yielded encouraging results. The transrectal US probe consisted of a 128element convex transducer array, reaching an effective imaging field of view of $170^{\circ}$. PAI showed enhanced capability in visualizing the blood-rich tumors in vivo, comparing to pure US imaging.

However, phantom and simulation studies have revealed limitations of transrectal light delivery. ${ }^{55,59,60}$ In transrectal light delivery, the light must penetrate through the rectum wall before it reaches the prostate. At the wavelength of $1000 \mathrm{~nm}$, the rectal absorption and scattering coefficients, $\mu_{a}$ and $\mu_{s}$, are greater than those in the prostate by factors of 2 and 5, respectively. ${ }^{55,66,67}$ Consequently, transrectal illumination suffers from significant energy loss due to the severe light scattering in the rectum wall and is not ideal for in vivo wholeprostate imaging (Figs. 2(d) and 2(h)). In addition, as demonstrated by Tang et al. ${ }^{60}$ and Peng et al. ${ }^{59}$ the prostate is asymmetrically illuminated under transrectal light delivery, which largely favors the detection of tumors located proximal to the rectum. To achieve whole-prostate imaging, a more uniformly distributed illumination is desired.

\subsection{Transurethral light delivery}

Unlike the rectum, which is located to the posterior of the prostate, the urethra passes through the center of the prostate; therefore, transurethral light 

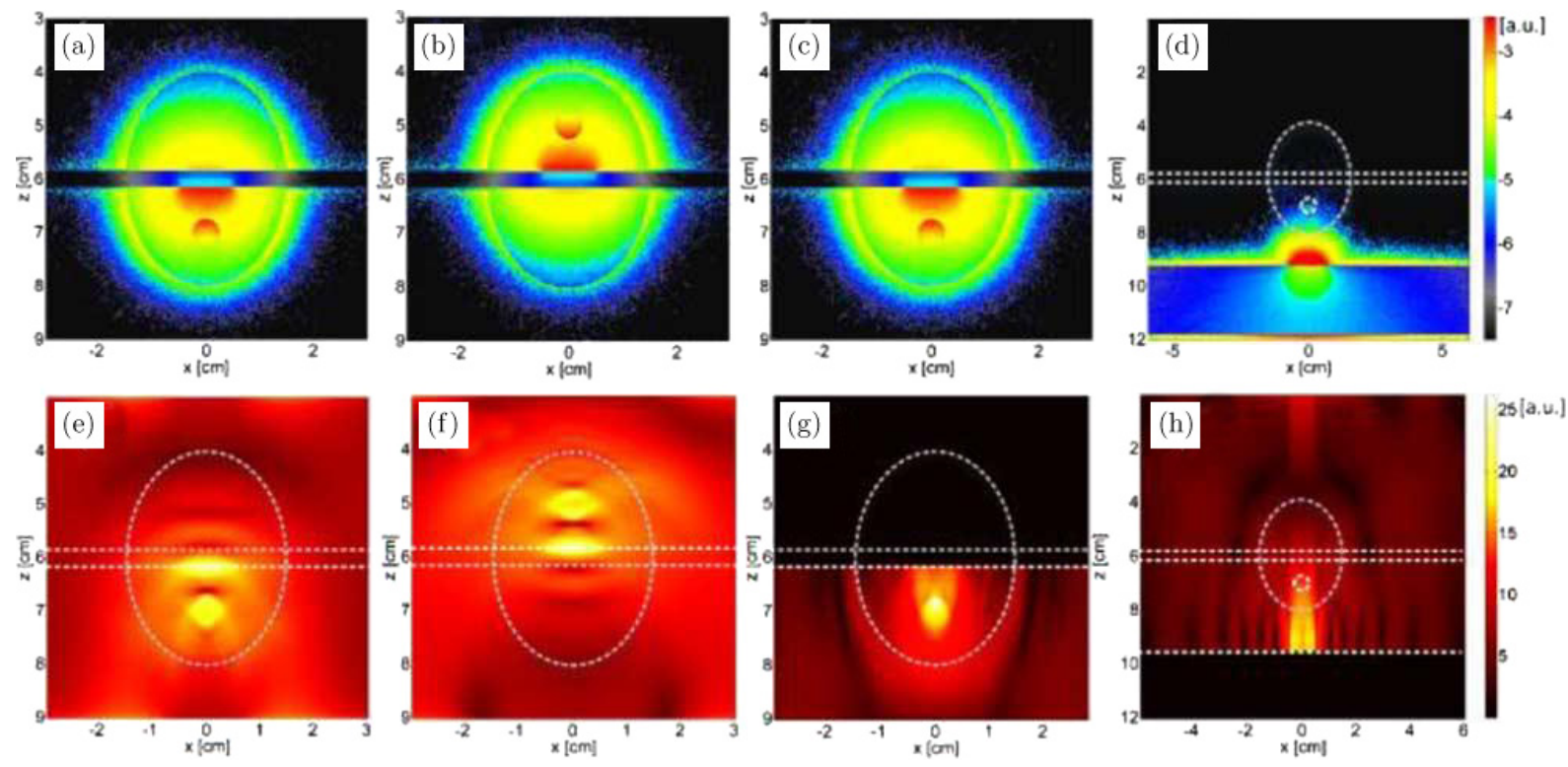

Source: ( 2016 IEEE. Reproduced, with permission, from Refs. 60.

Fig. 2. Monte Carlo simulation results of PAI of tumor-imbedded prostate models at different imaging configurations $(\lambda=763 \mathrm{~nm})$. (a) and (e) are the energy deposition and the PA image, respectively, using a transurethral light source and transrectal US detection. (b) and (f) are under the same configuration as (a) and (e) but with the tumor in the top half of the prostate. (c) and (g) are the energy deposition and PA image, respectively, using a transurethral light source and US detection. (d) and $(\mathrm{h})$ are the energy deposition and PA image, respectively, using a transrectal light source and US detection. The white dash lines indicate the positions of the urethra, prostate and tumor in the PA images.

delivery enables symmetric illumination of the prostate, although the half of the prostate closer to the rectum is slightly favored over the other (Figs. 2(a), 2(b), 2(e), and 2(f)). ${ }^{60}$ The $\mu_{a}$ and $\mu_{s}$ of the urethra wall are much lower than the rectum wall, thus only causing minimal energy loss. ${ }^{55,57,60}$ A phantom study by Yaseen et al. has shown that in transurethral light delivery, increasing light attenuation, as the distance from the light source increases, is compensated by the increasing proximity to the US transducer located in the rectum, whereas in transrectal light delivery, the light attenuation is not compensated for because the US transducer is located on the same side as the light source. ${ }^{57}$ Monte Carlo simulation studies that followed have also yielded a consensus; transurethral light delivery coupled with transrectal US probe is currently the optimal configuration for human prostate PAI. ${ }^{55,59,60}$

Based on the reported optimal configuration, PAI of the prostate inside an animal model further validated the feasibility of PAI for prostate imaging and therapy monitoring. ${ }^{61,68}$ Implemented by Bell et al., PAI of a canine prostate implanted with brachytherapy seeds using a transurethral light source and a transrectal US probe demonstrated excellent contrast and high signal-to-noise ratio, especially compared to conventional US imaging. ${ }^{61}$ A brachytherapy seed is a small, radioactive capsule that is permanently implanted in the prostate to treat the tumor. ${ }^{69}$ Transurethral light delivery was achieved by inserting the optical fiber via a catheter, which is a common clinical technique, thus does not increase the complexity of the process. In addition, a short-lag spatial coherence (SLSC) beamformer yielded better image quality than the traditional delay-and-sum (DAS) beamformer, owing to its higher sensitivity. ${ }^{70} \mathrm{PAI}$ demonstrates promising potential in aiding the implantation of brachytherapy seeds and monitoring the seeds inside the prostate.

Differing from MRI, PAI is capable of real-time imaging and is relatively inexpensive. PAI can thus potentially be employed to guide prostate biopsies and thus enable a fast, targeted prostate biopsy. Furthermore, if PAI can differentiate malignant tumor and $\mathrm{BPH}$, patients with $\mathrm{BPH}$ may avoid unnecessary biopsies. To demonstrate the clinical feasibility of onsite prostate cancer detection through PAI, more in vivo studies using transurethral light delivery are required. 


\section{PAI of Prostate Cancer Using Endogenous Contrast}

Because of its excellent optical absorption, protein hemoglobin in red blood cells is a major biomarker that enables label-free PAI of cancer. ${ }^{71}$ The capability of single blood vessel imaging allows for the detection and monitoring of excessive angiogenesis, ${ }^{72}$ which characterizes prostatic tumor development. ${ }^{73-75}$ Furthermore, tumor-related vasculature typically has different structural features compared to normal blood vessels, such as increased vessel tortuosity, vessel dilation, excessive branching, and necrosis. ${ }^{76,77}$ Therefore, the capture of abnormal distributions of red blood cells along with cancerrelated morphological features of blood vessels through PAI hold promising potential for the detection of prostatic tumors.

Wang et al. implemented in vivo and ex vivo $\mathrm{PAI}$ of a canine prostate with a pseudo cancerous lesion induced by the injection of blood, and demonstrated the unique ability of PAI in locating a blood-rich region in the prostate by comparing it with pure US imaging. ${ }^{58}$ In vivo US imaging provided good structural images of the canine prostate but failed to differentiate the induced blood-rich lesion, whereas PAI successfully illustrated the lesion. ${ }^{58}$ Furthermore, the matching increase of PA signal intensity and the amount of blood injected suggests that PAI has the potential to provide functional information, such as hemoglobin concentration, since PA signal amplitude is directly proportional to the optical energy absorbed by the object, which is directly associated with the concentration of the optical absorber ${ }^{78}$ Ex vivo PAI allows for the imaging of an excised prostate at a full $360^{\circ}$ imaging aperture through scanning, yielding high-resolution images that adequately illustrate the structure of the prostate and the urethra (Fig. 3(b)). ${ }^{58}$ However, in in vivo PAI of the prostate, the US transducer generally has a limited imaging aperture, which lowers the image quality (Figs. $3(\mathrm{c})-3(\mathrm{f})) .{ }^{58}$ This issue may be mitigated by the improvement of the transducer array, using advanced reconstructive algorithm, ${ }^{79,80}$ and physically moving the US transducer.

Bauer et al. demonstrated the ability of PAI for imaging blood vessels and monitoring tumor growth in mouse dorsal skin flaps implanted with PC-3 prostate tumor cells. ${ }^{81}$ Owing to the excellent optical absorption of red blood cells, PAI successfully illustrated angiogenesis and hemorrhage in the implanted tumors that were invisible under pure US imaging (Fig. 4). ${ }^{81}$ Compared with fluorescent imaging and US imaging, the distinctive feature of PAI is its ability to image the vascular network at a satisfactory resolution without utilizing contrast agents. ${ }^{81}$ Additionally, because US imaging provides good depth information and structural identification of the prostate, the coregistration of PA and US images can provide complementary information of tumor progression in a $2 \mathrm{D}$ and $3 \mathrm{D}$ manner. ${ }^{57,64,81}$

Oxyhemoglobin $\left(\mathrm{HbO}_{2}\right)$ and deoxygenated hemoglobin $(\mathrm{dHb})$ have different optical absorption properties at wavelengths within the range of PAI light sources. ${ }^{82}$ Capitalizing on this difference in absorption spectra, spectroscopic PAI enables the characterization of red blood cells and is capable of differentiating malignant, benign, and normal prostatic tissue. ${ }^{63,81,83,84}$ Ex vivo multispectral PAI of cancer-bearing prostate cryosections by Dogra et al. successfully differentiated malignant and nonmalignant human prostate tissue with excellent sensitivity $(81.3 \%)$ and specificity $(96.2 \%),{ }^{83}$ both higher than the current screening methods. ${ }^{8}$ Prostate cryosections from excised human prostates were imaged at four different wavelengths: $760 \mathrm{~nm}$, $850 \mathrm{~nm}, 930 \mathrm{~nm}$, and $970 \mathrm{~nm}$, each corresponding to the optimal light absorption of $\mathrm{dHb}, \mathrm{HbO}_{2}$, lipids, and water, respectively. ${ }^{83}$ From the PA images at these four wavelengths, chromophore PA images for each of the four optical absorbers were reconstructed through algorithm developed by Cox et $a l .{ }^{85}$ The results revealed significantly higher mean intensity of $\mathrm{PA}$ signals of $\mathrm{dHb}$ in malignant prostate tissue than in nonmalignant tissue (Fig. 5), indicating hypoxia, which is an important characteristic in many types of cancers, including prostate cancer. ${ }^{77,86}$ Moreover, benign prostatic tissue was further differentiated from normal prostatic tissue by its lower mean intensity at $970 \mathrm{~nm} .{ }^{83}$ Spectroscopic PAI demonstrated its potential as a powerful tool for prostate cancer diagnosis.

Besides spectroscopy, PAI using frequency domain analysis is an alternative method that may be capable of label-free prostate cancer diagnosis. Exploiting the spectrum of PA signal amplitude at a range of PA wave frequency, frequency domain analysis has shown potential in determining important parameters, such as the size and concentration, of the optical absorber and thus enabling 


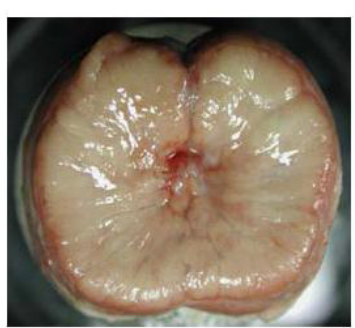

(a)
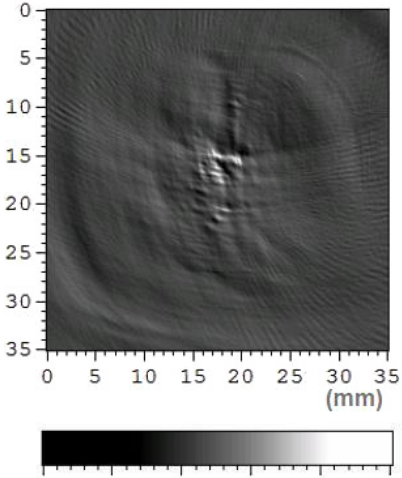

$\begin{array}{lllllllll}0.0 & 0.2 & 0.4 & 0.6 & 0.8 & 1.0\end{array}$

(d)

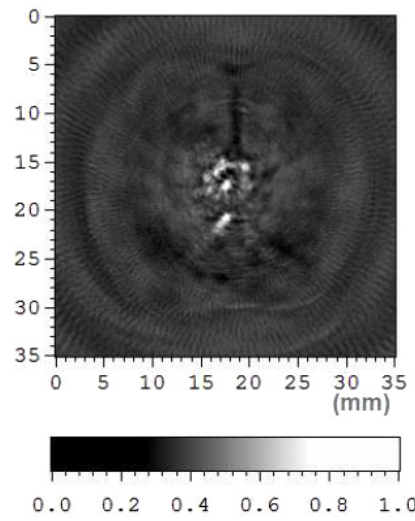

(b)
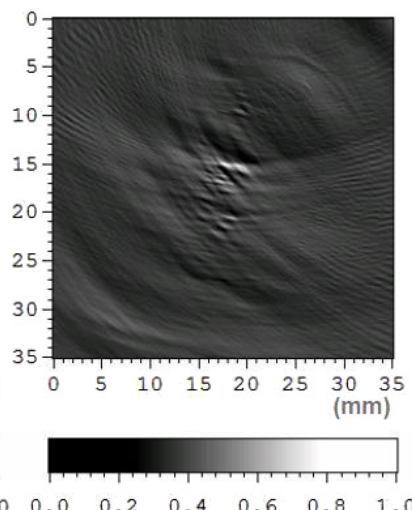

(e)

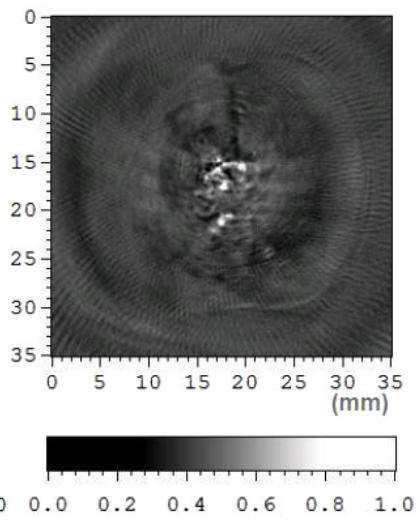

(c)

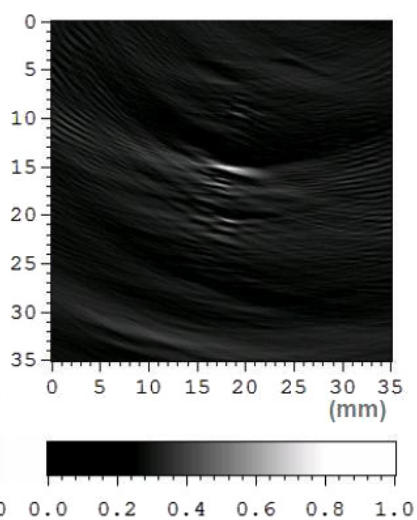

(f)

Source: () 2010 Optical Society of America. Reproduced, with permission, from Ref. 58.

Fig. 3. Ex vivo 2D photoacoustic tomography of an excised canine prostate. (a) Anatomic photography of the prostate crosssection; (b) PA image acquired with a detection view angle of $360^{\circ}$. The PA image adequately illustrates the structural outline of the prostate and the urethra in the center. (c)-(f) PA images acquired with detection view angles of $180^{\circ}, 90^{\circ}, 60^{\circ}$ and $30^{\circ}$. Wavelength of incident light $=710 \mathrm{~nm}$; pulse width $=5 \mathrm{~ns}$; pulse frequency $=10 \mathrm{~Hz}$.

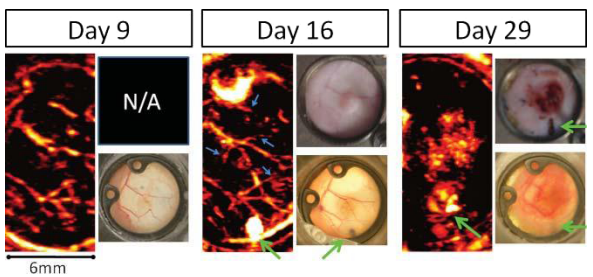

Source: (C) 2011 SPIE. Reproduced, with permission, form. Ref. 81.

Fig. 4. Optical and PA imaging of the progression of an implanted tumor in a mouse model. Photographs $(6 \times 6 \mathrm{~mm})$ of window chamber's skin side (top right) and coverslip side (bottom right) are shown alongside the PA image (left, $\lambda=800 \mathrm{~nm}, 6 \times 12 \mathrm{~mm}$ ). PA images on days 9 and 16 revealed a map and progression of the vascular network. The PA signal for day 29 is largely dominated by the extensive hemorrhage, which is consistent with the skin side photograph. Blood vessels are clearly resolved in the PA image on day 16 (labeled with small blue arrows), but not visualized in the skin side photograph. The large green arrows denote the fiducial ink mark. the quantitative characterization of the prostate tissue, as demonstrated by Kumon et al., Sinha et al., and Patterson et al. ${ }^{87-89}$ Several challenges, however, are faced in the development of both PAI spectroscopy and frequency domain analysis towards clinical implementation. The spectral parameters are likely to be different in an in vivo system, due to the overlying tissue; the algorithm for chromophore PA image reconstrucion is more complicated in reality, given that there are more components than $\mathrm{dHb}, \mathrm{HbO}_{2}$, lipids, and water in biological tissues. ${ }^{88,90}$ Additionally, in going from a $1 \mathrm{D}$ transducer to a $2 \mathrm{D}$ or $3 \mathrm{D}$ transducer array used in in vivo prostate imaging, the difficulty in image processing must be overcome. ${ }^{88}$

Although studies have shown effective endogenous contrast for tumor visualization, these biological 


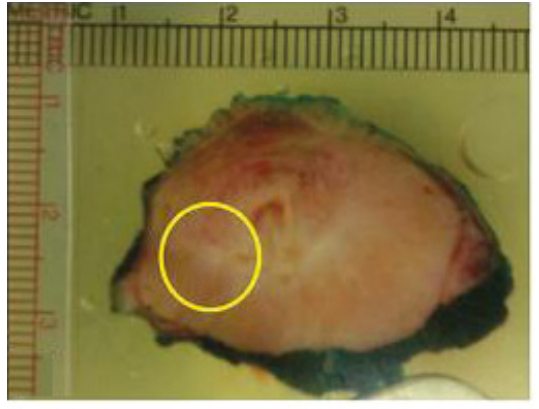

(a)

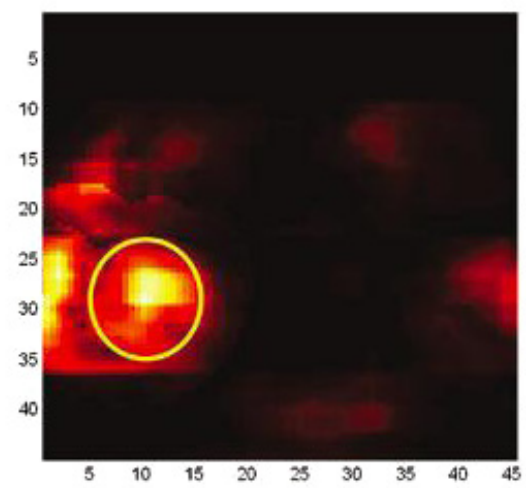

(c)

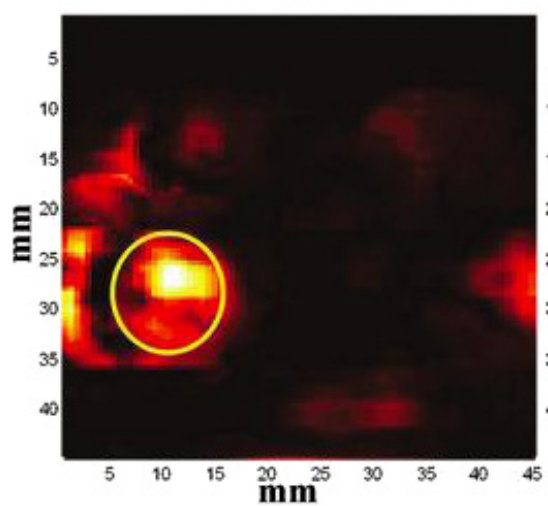

(e)

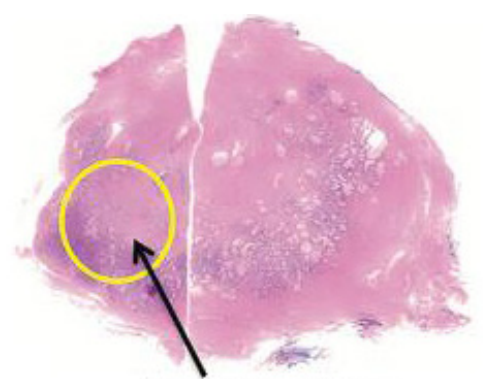

Malignant tissue

(b)

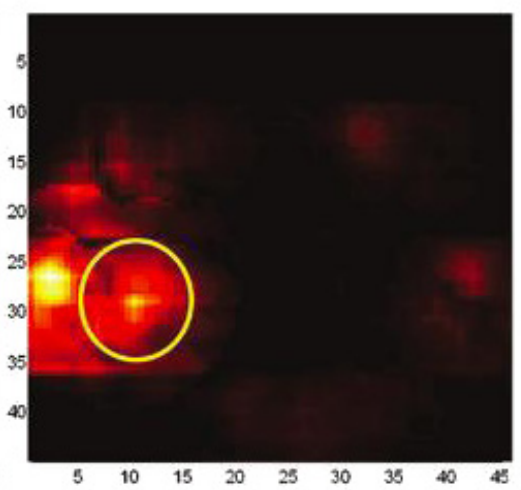

(d)

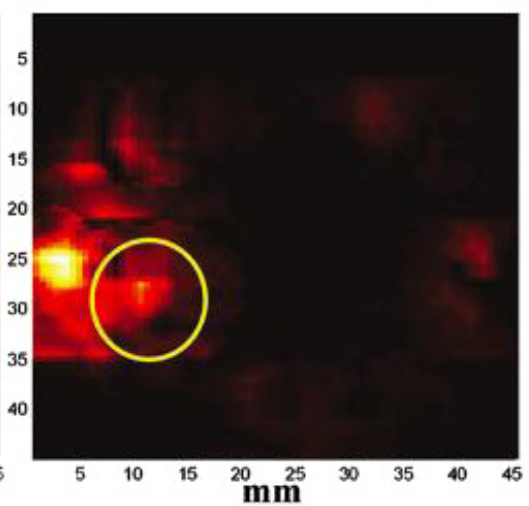

(f)

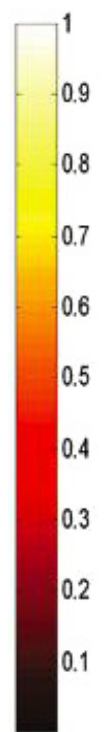

Source: () 2013 Journal of Clinical Imaging Science. Reproduced, with permission, from Ref. 83.

Fig. 5. Multispectral PA imgaes of a human prostate section. PA images are acquired at multiple laser wavelengths. Chromophore analysis was performed to extract PA images showing absorption of individual constituents from the multiwavelength images. (a) Photograph of imaged prostate specimen, (b) Histopathology slide of prostate with malignant region encircled, (c) Composite PA image acquired at $760 \mathrm{~nm}$ wavelength, (d) Composite PA image acquired at $850 \mathrm{~nm}$ wavelength, (e) Chromophore PA image showing the absorption of $\mathrm{dHb}$, and (f) Chromophore PA image showing the absorption of $\mathrm{HbO}_{2}$. Higher absorption of $\mathrm{dHb}$ was seen in the region of interest corresponding to malignant prostate tissue compared to $\mathrm{HbO}_{2}$.

constituents overall generate relatively weak PA signals; therefore, having a low signal-to-noise ratio may be a challenge when implemented in in vivo human prostate imaging. Prostate cancer may lack significant angiogenesis in its early stages, making the tumor difficult to visualize. More importantly, the aforementioned optical absorbers, such as $\mathrm{HbO}_{2}$ and $\mathrm{dHb}$, generally do not have a high degree of prostate cancer specificity. The imaging specificity and contrast of prostate cancer PAI can be improved, especially in the light of development toward clinical prostate cancer detection. 


\section{PAI of Prostate Cancer Using Exogenous Contrast Agents}

While natural biomarkers provide feasible endogenous contrast, the proper use of exogenous contrast agents, such as conjugated NIR dyes and conjugated gold nanoparticles (NPs), can further enhance PA signals of prostate imaging. ${ }^{91-94}$ Moreover, targeted imaging agents allow for prostate cancer-specific imaging through the effective binding of the targeting molecule to biomarkers that are specific for prostate cancer cells. ${ }^{91,92}$

Developed by Levi et al., a high-affinity, highstability prostate cancer PAI agent, AA3G-740, is capable of a nearly twofold increase in PA signals in mice in vivo. ${ }^{91}$ The imaging agent, AA3G-740, is essentially the highly stable fluorescent dye, ATTO740 , linked to the gastrin-releasing peptide (GRP), which binds specifically to the gastrin-releasing peptide receptor (GRPR). GRPR is commonly overexpressed in human prostate cancer cells (PC3), therefore allowing the targeted imaging of prostate cancer. ${ }^{95}$

Another important protein biomarker, prostatespecific membrane antigen (PSMA), is overexpressed on the surface of most prostate cancer cells. ${ }^{96}$ By attaching an NIR dye, IRDye800CW, to agents that specifically target PSMA, Dogra et al. have demonstrated that targeted PAI enables the study of prostate cancer cells at the molecular level. ${ }^{92}$ PSMA-targeting agents labeled with NIRabsorbing dyes allow for the recognition and differentiation of prostate cancer cells that express PSMA (C4-2) and the ones that do not (PC3). In addition to the NIR dye, the use of an acoustic lens also enhanced the PA signal by focusing the US waves at the transducer. ${ }^{97}$

In vivo prostate PAI using contrast agents often requires the protection of the contrast agents from unfavorable interaction with reactive biological constituents, such as enzymes, throughout the organism. Polyacrylamide (PAA) NPs developed by $\mathrm{Xu}$ et al. have demonstrated satisfactory ability in capsuling the contrast agent while targeting nucleolin, a molecule often overexpressed on cancer cells. ${ }^{94,98,99}$ Compared to US imaging, the in vivo PA images exhibited notably enhanced contrast of the tumors implanted in mice, whereas no change was observed in the pure US images (Fig. 6). ${ }^{94}$ Besides imaging, PAA NPs can also potentially serve as a cancer-treating drug delivery agent that

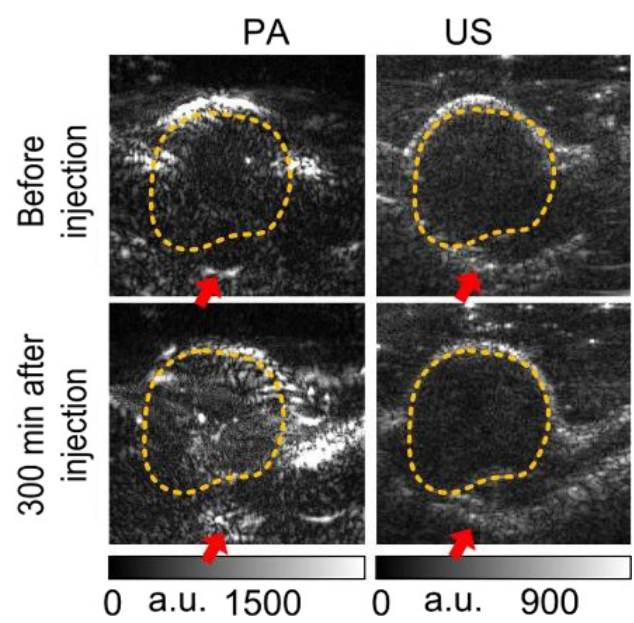

Source: (C) 2016 SPIE. Reproduced, with permission, from Ref. 94.

Fig. 6. In vivo PA images of a xenograft prostate tumor in rat before and after PAA NP injection (left), compared with US imaging (right). Dashed contour lines mark the tumor region; red arrows mark the blood vessels connecting to the tumor. The in vivo $\mathrm{PA}$ images exhibit enhanced contrast of the tumor, whereas no change can be observed in the pure US images.

simultaneously allows for the real-time monitoring of drug delivery through PAI. ${ }^{94}$

Contrast-agent-based PAI of prostate cancer possesses enhanced specificity and sensitivity, thus potentially allowing for the visualization of tumors with relatively minor angiogenesis. PAI also can provide important insights in the study of cancer cells at the cellular and molecular level through realtime monitoring. However, the effective binding of targeted agents to prostate cancer-specific biomarkers in larger living organisms remains a major challenge, and more in vivo animal testing is needed to examine the toxicity of the imaging agents as well as to estimate the effective dosage for human prostate imaging.

\section{Future Outlook}

PAI is growing rapidly as an innovative imaging modality for prostate cancer with minimal invasiveness. Technical improvement of the PAI instrumentation (e.g., the design of the US transducer array, optical and acoustic focusing, and the quality of the light source) may broaden its utility and propel its translation toward clinical application. In addition, clinical trials currently undertaken by Gambhir et al. using a transrectal PAI probe may provide helpful insights in the clinical feasibility of transrectal US detection and light delivery. ${ }^{100}$ 
Other possible applications of PAI include aiding prostate cancer therapy and enabling PAI-guided drug delivery. ${ }^{62,101-104}$ Real-time PAI can potentially provide helpful insights in the delivery of cancer-treating drugs by monitoring the tumor progression as well as tracking the drug distribution and concentration. ${ }^{101}$ Similarly, photonic-based cancer therapies, such as laser induced photodynamic therapy (PDT) ${ }^{105,106}$ and photothermal therapy (PTT), ${ }^{107}$ can also benefit from PAI, as PAI can monitor the drug distribution simultaneously during the therapy without the use of additional contrast agents. Furthermore, PAI can be combined with other imaging modalities, such as fluorescence imaging ${ }^{81,92,108}$ and US imaging, ${ }^{57,58,64,81,109}$ to provide complementary information and optimal image quality. Through PAI, minimally invasive onsite prostate cancer diagnosis with enhanced sensitivity and specificity may be achieved.

\section{Conclusion}

PAI has shown promising potential in overcoming the current bottleneck in prostate cancer detection, which is the difficulty in prostate cancer visualization. Because PAI has the potential to define cancerous regions and differentiate $\mathrm{BPH}$ from malignant tissue, targeted prostate biopsies may be enabled and unnecessary prostatectomies may be best avoided, minimizing patients' pain and cost. To overcome the penetration limit of PAI systems, transurethral light delivery coupled with a transrectal US detector offers optimal prostate imaging quality, although further instrumental improvement may also enable transurethral US detection. The different optical absorption properties of endogenous contrast sources, such as $\mathrm{HbO}_{2}$ and $\mathrm{dHb}$, may enable label-free prostate cancer detection because excessive angiogenesis is a typical characteristic of prostate cancer. The image quality and specificity of prostate cancer PAI can be further improved by using targeted exogenous contrast agents, which can also assist the molecular study of prostate cancer cells. As an emerging imaging modality, PAI may be capable of improving the sensitivity of prostate cancer detection, enabling PAI-guided prostate biopsy and cancer treatment, as well as advancing the study of prostate cancer cells.

\section{Acknowledgments}

The authors gratefully acknowledge the University of Oklahoma Research Council and the College of Engineering at the University of Oklahoma. This work was supported in part by the University of Oklahoma under the IBEST-OUHSC Grant. The authors would like to thank Pratik Samant for proofreading the paper.

\section{References}

1. Cancer Stat Facts: Prostate Cancer. National Cancer Institute (2015). available at: https:// seer.cancer.gov/statfacts/html/prost.html. [Last accessed on 2017 Mar 5].

2. D. Ilic, M. M. Neuberger, M. Djulbegovic, P. Dahm, "Screening for prostate cancer", Cochrane Database Syst. Rev. Jan. 31(3), CD004720 (2013).

3. A. A. A. Elgamal, H. P. Van Poppel, W. M. Van de Voorde, J. A. Van Dorpe, R. H. Oyen, L. V. Baert, "Impalpable invisible stage T1c prostate cancer: Characteristics and clinical relevance in 100 radical prostatectomy specimens-A different view", $J$. Urol. 157(1), 244-250 (1997).

4. T. H. Van der Kwast, "The trade-off between sensitivity and specificity of clinical protocols for identification of insignificant prostate cancer," Eur. Urol. 62(3), 469-471 (2012).

5. E. W. Steyerberg, M. J. Roobol, M. W. Kattan, T. H. van der Kwast, H. J. de Koning, F. H. Schroder, "Prediction of indolent prostate cancer: Validation and updating of a prognostic nomogram," J. Urol. 177(1), 107-112 (2007).

6. H. G. Welch, L. M. Schwartz, S. Woloshin, "Prostate-specific antigen levels in the United States: Implications of various definitions for abnormal," J. Natl. Cancer Inst. 97(15), 11321137 (2005).

7. R. C. Flanigan, W. J. Catalona, J. P. Richie, F. R. Ahmann, M. A. Hudson, P. T. Scardino, J. B. Dekernion, T. L. Ratliff, L. R. Kavoussi, B. L. Dalkin et al., "Accuracy of digital rectal examination and transrectal ultrasonography in localizing prostate-cancer," J. Urol. 152(5), 1506-1509 (1994).

8. E. D. Cook, A. C. Nelson, "Prostate cancer screenin," Curr. Oncol. Rep. 13 (1), 57-62 (2011).

9. W. J. Catalona, J. P. Richie, F. R. Ahmann, M. A. Hudson, P. T. Scardino, R. C. Flanigan, J. B. DeKernion, T. L. Ratliff, L. R. Kavoussi, B. L. Dalkin et al., "Comparison of digital rectal examination and serum prostate specific antigen in the early detection of prostate cancer: Results of a 
multicenter clinical trial of 6,630 Men," J. Urol. 197 (2S), S200-S207 (2017).

10. T. A. Stamey, N. Yang, A. R. Hay, J. E. McNeal, F. S. Freiha, E. Redwine, "Prostate-specific antigen as a serum marker for adenocarcinoma of the prostate," N. Engl. J. Med. 317(15), 909-916 (1987).

11. P. Tenke, J. Horti, P. Balint, B. Kovacs, Prostate cancer screening, Prostate Cancer, J. Ramon, L. J. Denis, Eds., pp. 65-81, Springer, Berlin, Heidelberg (2007).

12. R. Postma, F. H. Schroder, G. J. van Leenders, R. F. Hoedemaeker, A. N. Vis, M. J. Roobol, T. H. van der Kwast, "Cancer detection and cancer characteristics in the European Randomized Study of Screening for Prostate Cancer (ERSPC) Section Rotterdam: A comparison of two rounds of screening," Eur. Urol. 52(1), 89-97 (2007).

13. H. M. Nagler, E. W. Gerber, P. Homel, J. R. Wagner, J. Norton, S. Lebovitch, J. L. Phillips, "Digital rectal examination is barrier to populationbased prostate cancer screenin," Urology 65(6), 1137-1140 (2005).

14. G. S. Gerber, I. M. Thompson, R. Thisted, G. W. Chodak, "Disease-specific survival following routine prostate cancer screening by digital rectal examination," J. Am. Med. Assoc. 269(1), 61-64 (1993).

15. American cancer society: What tests can detect prostate cancer early? American Cancer Society (2016), available at https://www.cancer.org/cancer/prostate-cancer/early-detection/tests.html.

16. T. A. Stamey, F. S. Freiha, J. E. McNeal, E. A. Redwine, A. S. Whittemore, H.-P. Schmid, "Localized prostate cancer: Relationship of tumor volume to clinical significance for treatment of prostate cancer," Cancer 71(S3), 933-938 (1993).

17. K. T. McVary, C. G. Roehrborn, A. L. Avins, M. J. Barry, R. C. Bruskewitz, R. F. Donnell, H. E. Foster, Jr., C. M. Gonzalez, S. A. Kaplan, D. F. Penson et al., "Update on AUA guideline on the management of benign prostatic hyperplasia," J. Urol. 185 (5), 1793-1803 (2011).

18. R. J. Babaian, C. Mettlin, R. Kane, G. P. Murphy, F. Lee, J. R. Drago, A. Chesley, "The relationship of prostate-specific antigen to digital rectal examination and transrectal ultrasonography: Findings of the American cancer society national prostate cancer detection project," Cancer 69(5), 1195-1200 (1992).

19. C. J. Harvey, J. Pilcher, J. Richenberg, U. Patel, F. Frauscher, "Applications of transrectal ultrasound in prostate cancer," Br. J. Radiol. 85(1), S3-S17 (2012).

20. W. J. Ellis, M. K. Brawer, "The significance of isoechoic prostatic carcinoma," J. Urol. 152(6II), 2304-2307 (1994).
21. F. A. Ganie, M. S. Wanie, S. A. Ganie, H. Lone, M. Gani, M. F. Mir, N. A. Khan, "Correlation of transrectal ultrasonographic findings with histo pathology in prostatic cancer," J. Educ. Health Promot. 3, 38 (2014).

22. P. J. Littrup, S. E. Bailey, "Prostate cancer: The role of transrectal ultrasound and its impact on cancer detection and management," Radiol. Clin. North Am. 38(1), 87-113 (2000).

23. F. J. Fowler, Jr., M. J. Barry, B. Walker-Corkery, J. F. Caubet, D. W. Bates, J. M. Lee, A. Hauser, M. McNaughton-Collins, "The impact of a suspicious prostate biopsy on patients' psychological, socio-behavioral, and medical care outcomes," $J$. Gen. Intern. Med. 21(7), 715-721 (2006).

24. S. Loeb, A. Vellekoop, H. U. Ahmed, J. Catto, M. Emberton, R. Nam, D. J. Rosario, V. Scattoni, Y. Lotan, "Systematic review of complications of prostate biopsy," Eur. Urol. 64 (6), 876-892 (2013).

25. T. H. Ecke, S. Gunia, P. Bartel, S. Hallmann, S. Koch, J. Ruttloff, "Complications and risk factors of transrectal ultrasound guided needle biopsies of the prostate evaluated by questionnaire," Urol. Oncol. 26(5), 474-478 (2008).

26. J. H. Yacoub, S. Verma, J. S. Moulton, S. Eggener, A. Oto, "Imaging-guided prostate biopsy: Conventional and emerging techniques," Radiographics 32(3), 819-837 (2012).

27. G. C. Durkan, D. R. Greene, "Diagnostic dilemmas in detection of prostate cancer in patients undergoing transrectal ultrasound-guided needle biopsy of the prostate," Prostate Cancer Prostatic Dis. 3(1), 13-20 (2000).

28. B. O. B. Djavan, A. Zlotta, M. Remzi, K. Ghawidel, A. L. I. Basharkhah, C. C. Schulman, M. Marberger, "Optimal predictors of prostate cancer on repeat prostate biopsy: A prospective study of 1,051 men," J. Urol. 163(4), 1144-1149 (2000).

29. O. Ukimura, O. Durrani, R. J. Babaian, "Role of psa and its indices in determining the need for repeat prostate biopsies," Urology 50(1), 66-72 (1997).

30. T. O. Morgan, D. G. McLeod, E. S. Leifer, G. P. Murphy, J. W. Moul, "Prospective use of free prostate-specific antigen to avoid repeat prostate biopsies in men with elevated total prostate-specific antigen," Urology 48(6, Supplement 1), 76-80 (1996).

31. A. R. Padhani, C. J. Gapinski, D. A. Macvicar, G. J. Parker, J. Suckling, P. B. Revell, M. O. Leach, D. P. Dearnaley, J. E. Husband, "Dynamic contrast enhanced MRI of prostate cancer: Correlation with morphology and tumour stage, histological grade and PSA," Clin. Radiol. 55(2), 99-109 (2000). 
32. A. P. Kirkham, M. Emberton, C. Allen, "How good is MRI at detecting and characterising cancer within the prostate?" Eur. Urol. 50(6), 1163-1174 (2006).

33. L. Dickinson, H. U. Ahmed, C. Allen, J. O. Barentsz, B. Carey, J. J. Futterer, S. W. Heijmink, P. J. Hoskin, A. Kirkham, A. R. Padhani et al., "Magnetic resonance imaging for the detection, localisation, and characterisation of prostate cancer: Recommendations from a European consensus meeting", Eur. Urol. 59(4), 477-494 (2011).

34. T. Hara, N. Kosaka, H. Kishi, "PET imaging of prostate cancer using carbon-11-choline," J. Nucl. Med. 39(6), 990-995 (1998).

35. C. Spick, K. Herrmann, J. Czernin, "Evaluation of prostate cancer with 11C-Acetate PET/CT," J. Nucl Med. 57(Suppl 3), 30S-37S (2016).

36. M. Picchio, M. Piert, "Prostate cancer imaging," Eur. J. Nucl. Med. Mol. Imaging 40(Suppl 1), S1-S4 (2013).

37. M. Xu, L. V. Wang, "Photoacoustic imaging in biomedicine," Rev. Sci. Instrum. 77(4), 041101 (2006).

38. L. V. Wang, S. Hu, "Photoacoustic tomography: In vivo imaging from organelles to organs," Science 335(6075), 1458-1462 (2012).

39. P. Beard, "Biomedical photoacoustic imaging," Interface Focus 1(4), 602-631 (2011).

40. C. G. A. Hoelen, F. F. M. de Mul, R. Pongers, A. Dekker, "Three-dimensional photoacoustic imaging of blood vessels in tissue," Opt. Lett. 23(8), 648-650 (1998).

41. H. Wang, X. Yang, Y. Liu, B. Jiang, Q. Luo, "Reflection-mode optical-resolution photoacoustic microscopy based on a reflective objective," Opt. Express 21(20), 24210-24218 (2013).

42. H. Wang, X. Yang, Z. Wang, Z. Deng, H. Gong, Q. Luo, "Early monitoring of cerebral hypoperfusion in rats by laser speckle imaging and functional photoacoustic microscopy," J. Biomed. Opt. 17(6), 0612071-0612079 (2012).

43. Y. Liu, X. Yang, H. Gong, B. Jiang, H. Wang, G. $\mathrm{Xu}, \mathrm{Y}$. Deng, "Assessing the effects of norepinephrine on single cerebral microvessels using optical-resolution photoacoustic microscope," J. Biomed. Opt. 18(7), 076007-076007 (2013).

44. X. Ji, K. Xiong, S. Yang, D. Xing, "Intravascular confocal photoacoustic endoscope with dual-element ultrasonic transducer," Opt. Express 23(7), 9130-9136 (2015).

45. L. Xiang, B. Wang, L. Ji, H. Jiang, "4-D photoacoustic tomography," Sci. Rep. 3, 1113 (2013).

46. Y. Lao, D. Xing, S. Yang, L. Xiang, "Noninvasive photoacoustic imaging of the developing vasculature during early tumor growth," Phys. Med. Biol. 53(15), 4203-4212 (2008).

47. G. J. Tserevelakis, I. Vrouvaki, P. Siozos, K. Melessanaki, K. Hatzigiannakis, C. Fotakis, G. Zacharakis, "Photoacoustic imaging reveals hidden underdrawings in paintings," Sci. Rep. 7(1), 747 (2017).

48. A. G. Bell, "On the production and reproduction of sound by light," Am. J. Sci. 118, 305-324 (1880).

49. J. Xia, J. Yao, L. V. Wang, "Photoacoustic tomography: Principles and advances," Electromagn. Waves (Camb.) 147, 1-22 (2014).

50. T. Durduran, R. Choe, W. B. Baker, A. G. Yodh, "Diffuse optics for tissue monitoring and tomography," Rep. Prog. Phys. 73(7), 076701 (2010).

51. R. S. C. Cobbold, Foundations of Biomedical Ultrasound, Oxford University Press, Oxford (2006).

52. P. J. A. Frinking, A. Bouakaz, J. Kirkhorn, F. J. Ten Cate, N. de Jong, "Ultrasound contrast imaging: Current and new potential methods," Ultrasound Med. Biol. 26(6), 965-975 (2000).

53. S. Hu, K. Maslov, L. V. Wang, "Second-generation optical-resolution photoacoustic microscopy with improved sensitivity and speed," Opt. Lett. 36(7), 1134-1136 (2011).

54. J. J. Niederhauser, M. Jaeger, R. Lemor, P. Weber, M. Frenz, "Combined ultrasound and optoacoustic system for real-time high-contrast vascular imaging in vivo," IEEE Trans. Med. Imaging 24(4), 436-440 (2005).

55. S. H. El-Gohary, M. K. Metwally, S. Eom, S. H. Jeon, K. M. Byun, T.-S. Kim, "Design study on photoacoustic probe to detect prostate cancer using 3D Monte Carlo simulation and finite element method," Biomed. Eng. Lett. 4(3), 250-257 (2014).

56. C. H. Lee, O. Akin-Olugbade, A. Kirschenbaum, "Overview of prostate anatomy, histology, and pathology," Endocrinol. Metab. Clin. North Am. 40(3), 565-575 (2011).

57. M. A. Yaseen, S. A. Ermilov, H. P. Brecht, R. Su, A. Conjusteau, M. Fronheiser, B. A. Bell, M. Motamedi, A. A. Oraevsky, "Optoacoustic imaging of the prostate: Development toward image-guided biopsy," J. Biomed. Opt. 15(2), 021310 (2010).

58. X. D. Wang, W. W. Roberts, P. L. Carson, D. P. Wood, J. B. Fowlkes, "Photoacoustic tomography: A potential new tool for prostate cancer," Biomed. Opt. Express 1(4), 1117-1126 (2010).

59. P. Dong-qing, P. Yuan-yuan, G. Jian, L. Hui, "Laser illumination modality of photoacoustic imaging technique for prostate cancer," J. Phys. Conf. Ser. 679(1), 012026 (2016). 
60. S. Tang, J. Chen, P. Samant, K. Stratton, L. Xiang, "Transurethral photoacoustic endoscopy for prostate cancer: A simulation study," IEEE. Trans. Med. Imaging 35(7), 1780-1787 (2016).

61. M. A. L. Bell, X. Y. Guo, D. Y. Song, E. M. Boctor, "Transurethral light delivery for prostate photoacoustic imaging," J. Biomed. Opt. 20(3) 036002 (2015).

62. M. A. Lediju Bell, N. P. Kuo, D. Y. Song, J. Kang, E. M. Boctor, In vivo photoacoustic imaging of prostate brachytherapy seeds. Proc. SPIE Photons Plus Ultrasound: Imaging and Sensing 2014, A. A. Oraevsky, L. V. Wang, Eds., pp. 894348-494310, San Francisco, CA, USA (2014).

63. T. Mitcham, K. Dextraze, H. Taghavi, M. Melancon, R. Bouchard, "Photoacoustic imaging driven by an interstitial irradiation source," Photoacoustics 3(2), 45-54 (2015).

64. A. Horiguchi, K. Tsujita, K. Irisawa, T. Kasamatsu, K. Hirota, M. Kawaguchi, M. Shinchi, K. Ito, T. Asano, H. Shinmoto et al., "A pilot study of photoacoustic imaging system for improved realtime visualization of neurovascular bundle during radical prostatectomy," Prostate $\mathbf{7 6}(3)$, 307-315 (2016).

65. M. Ishihara, M. Shinchi, A. Horiguchi, H. Shinmoto, H. Tsuda, K. Irisawa, T. Wada, T. Asano, "Possibility of transrectal photoacoustic imagingguided biopsy for detection of prostate cancer," Proc. SPIE Photons Plus Ultrasound: Imaging and Sensing 2017, A. A. Oraevsky, L. V. Wang, Eds., pp. 100642U-100645, San Francisco, CA, USA (2017).

66. P. D. Agrba, M. Y. Kirillin, A. I. Abelevich, V. A. Kamensky, Mechanical compression for biotissue image enhancement in optical coherence tomography, Proc SPIE Saratov Fall Meeting 2009: International School for Junior Scientists and Students on Optics, Laser Physics, and Biophotonics, V. V. Tuchin, E. A. Genina, Eds., p. 754703, Saratov, Russian Federation (2009).

67. J. Mobley, T. Vo-Dinh, "Optical properties of tissue," Biomedical Photonics Handbook, CRC Press, Chapter 2.4, (2003), pp. 77-110.

68. M. A. L. Bell, N. P. Kuo, D. Y. Song, J. U. Kang, E. M. Boctor, "In vivo visualization of prostate brachytherapy seeds with photoacoustic imaging," J. Biomed. Opt. 19(12), 126011 (2014).

69. E. J. Kaplan, Brachytherapy seed, Google Patents, Patents publication \#:US6746661B2. Retrieved from https://google.com/patents/US6746661, (2004).

70. M. A. L. Bell, N. Kuo, D. Y. Song, E. M. Boctor, "Short-lag spatial coherence beamforming of photoacoustic images for enhanced visualization of prostate brachytherapy seeds," Biomed. Opt. Express 4(10), 1964-1977 (2013).

71. D. J. Faber, E. G. Mik, M. C. G. Aalders, T. G. van Leeuwen, "Light absorption of (oxy-)hemoglobin assessed by spectroscopic optical coherence tomography," Opt. Lett. 28(16), 1436-1438 (2003).

72. J. Folkman, Angiogenesis, Biology of Endothelial Cells, E. A. Jaffe, Ed., pp. 421-428, Springer, Boston, US (1984).

73. W. J. Huss, C. F. Hanrahan, R. J. Barrios, J. W. Simons, N. M. Greenberg, "Angiogenesis and prostate cancer: Identification of a molecular progression switch," Cancer Res. 61(6), 2736-2743 (2001).

74. S. S. Chang, D. S. O'Keefe, D. J. Bacich, V. E. Reuter, W. D. W. Heston, P. B. Gaudin, "Prostate-specific membrane antigen is produced in tumor-associated neovasculatur," Clin. Cancer Res. 5(10), 2674-2681 (1999).

75. M. K. Brawer, R. E. Deering, M. Brown, S. D. Preston, S. A. Bigler, "Predictors of pathologic stage in prostatic carcinoma: The role of neovascularity," Cancer 73(3), 678-687 (1994).

76. P. Carmeliet, R. K. Jain, "Angiogenesis in cancer and other diseases," Nature 407 (6801), 249-257 (2000).

77. M. W. Dewhirst, E. J. Ozimek, J. Gross, T. C. Cetas, "Will hyperthermia conquer the elusive hypoxic cell? Implications of heat effects on tumor and normal-tissue microcirculation," Radiology 137(3), 811-817 (1980).

78. L. V. Wang, H.-I. Wu, Photoacoustic tomography, Biomedical Optics, pp. 283-321, John Wiley \& Sons, (2009).

79. D. Yang, D. Xing, Limited-view scanning photoacoustic imaging based on algebraic reconstruction techniques, Fourth Int. Conf. Photonics and Imaging in Biology and Medicine, K. Xu, Q. Luo, D. Xing, A. V. Priezzhev, V. V. Tuchin, Eds., p. 604701, Tianjin china (2005).

80. J. Gamelin, A. Aguirre, A. Maurudis, L. V. Wang, Q. Zhu, Improvements in time resolution of tomographic photoacoustic imaging using a priori information for multiplexed systems, Proc. SPIE Photons Plus Ultrasound: Imaging and Sensing 2009, A. A. Oraevsky, L. V. Wang, Eds., pp. 71771C-71712, SanJose, CA (2009).

81. D. R. Bauer, R. Olafsson, L. G. Montilla, R. S. Witte, "3-D photoacoustic and pulse echo imaging of prostate tumor progression in the mouse window chamber," J. Biomed. Opt. 16(2), 026012 (2011).

82. A. Roggan, M. Friebel, K. Dorschel, A. Hahn, G. Muller, "Optical properties of circulating human blood in the wavelength range 400-2500 nm," $J$. Biomed. Opt. 4(1), 36-46 (1999). 
83. V. S. Dogra, B. K. Chinni, K. S. Valluru, J. V. Joseph, A. Ghazi, J. L. Yao, K. Evans, E. M. Messing, N. A. Rao, "Multispectral photoacoustic imaging of prostate cancer: Preliminary ex vivo results," J. Clin. Imaging Sci. 3, 41 (2013).

84. R. E. Kumon, C. X. Deng, X. Wang, Spectrum analysis of photoacoustic imaging data from prostate adenocarcinoma tumors in a murine model, Proc SPIE Photons Plus Ultrasound: Imaging and Sensing 2011, A. A. Oraevsky, L. V. Wang, Eds., pp. 78993Q-78996, San Francisco, CA, USA (2011).

85. B. T. Cox, S. R. Arridge, P. C. Beard, "Estimating chromophore distributions from multiwavelength photoacoustic images," J. Opt. Soc. Am. A 26(2), 443-455 (2009).

86. M. Milosevic, P. Chung, C. Parker, R. Bristow, A. Toi, T. Panzarella, P. Warde, C. Catton, C. Menard, A. Bayley et al., "Androgen withdrawal in patients reduces prostate cancer hypoxia: Implications for disease progression and radiation response," Cancer Res. 67(13), 6022-6025 (2007).

87. R. E. Kumon, C. X. Deng, X. Wang, "Frequencydomain analysis of photoacoustic imaging data from prostate adenocarcinoma tumors in a murine model," Ultrasound Med. Biol. 37(5), 834-839 (2011).

88. S. Sinha, N. A. Rao, B. K. Chinni, V. S. Dogra, "Evaluation of frequency domain analysis of a multiwavelength photoacoustic signal for differentiating malignant from benign and normal prostates: Ex vivo study with human prostates," J. Ultrasound Med. 35(10), 2165-2177 (2016).

89. M. P. Patterson, C. B. Riley, M. C. Kolios, W. M. Whelan, "Optoacoustic characterization of prostate cancer in an in vivo transgenic murine model," J. Biomed. Opt. 19(5) 056008 (2014).

90. B. Cox, J. G. Laufer, S. R. Arridge, P. C. Beard, "Quantitative spectroscopic photoacoustic imaging: A review," J. Biomed. Opt. 17(6), 061202106120222 (2012).

91. J. Levi, A. Sathirachinda, S. S. Gambhir, "A highaffinity, high-stability photoacoustic agent for imaging gastrin-releasing peptide receptor in prostate cancer," Clin. Cancer Res. 20(14), 3721-3729 (2014).

92. V. Dogra, B. Chinni, S. Singh, H. Schmitthenner, N. Rao, J. J. Krolewski, K. L. Nastiuk, "Photoacoustic imaging with an acoustic lens detects prostate cancer cells labeled with PSMA-targeting nearinfrared dye-conjugates," J. Biomed. Opt. 21(6), 66019 (2016).

93. A. Agarwal, S. W. Huang, M. O'Donnell, K. C. Day, M. Day, N. Kotov, S. Ashkenazi, "Targeted gold nanorod contrast agent for prostate cancer detection by photoacoustic imaging," J. Appl. Phys. 102(6), 064701 (2007).

94. G. Xu, M. Qin, A. Mukundan, J. Siddiqui, M. Takada, P. Vilar-Saavedra, S. A. Tomlins, R. Kopelman, X. Wang, Prostate cancer characterization by optical contrast enhanced photoacoustics. Proc SPIE Photons Plus Ultrasound: Imaging and Sensing 2016, A. A. Oraevsky, L. V. Wang, Eds., pp. 97080I-97086, San Francisco, CA, USA (2016).

95. R. Markwalder, J. C. Reubi, "Gastrin-releasing peptide receptors in the human prostate: Relation to neoplastic transformation," Cancer Res. 59(5), 1152-1159 (1999).

96. A. Ghosh, W. D. W. Heston, "Tumor target prostate specific membrane antigen (PSMA) and its regulation in prostate cancer," J. Cell. Biochem. 91(3), 528-539 (2004).

97. N. A. Rao, D. Lai, S. Bhatt, S. C. Arnold, B. Chinni, V. S. Dogra, "Acoustic lens characterization for ultrasound and photoacoustic C-scan imaging modalities," Conf. Proc. IEEE Eng. Med. Biol. Soc. 2008, 2177-2180 (2008).

98. M. Derenzini, V. Sirri, D. Trere, R. L. Ochs, "The quantity of nucleolar proteins nucleolin and protein B23 is related to cell doubling time in human cancer cells," Lab. Invest. 73(4), 497-502 (1995).

99. Y. Otake, S. Soundararajan, T. K. Sengupta, E. A. Kio, J. C. Smith, M. Pineda-Roman, R. K. Stuart, E. K. Spicer, D. J. Fernandes, "Overexpression of nucleolin in chronic lymphocytic leukemia cells induces stabilization of bcl-2 mRNA," Blood, 109(7), 3069-75 (2006).

100. S. S. Gambhir, S. R. Kothapalli, Photoacoustic Imaging (PAI) of the Prostate: A Clinical Feasibility Study. ClincialTrails.gov (2014), Retrieved from https://clinicaltrials.gov/show/NCT01551576.

101. J. Zhong, L. Wen, S. Yang, L. Xiang, Q. Chen, D. Xing, "Imaging-guided high-efficient photoacoustic tumor therapy with targeting gold nanorods," Nanomedicine 11(6), 1499-1509 (2015).

102. J. Xia, C. Kim, J. F. Lovell, "Opportunities for photoacoustic-guided drug delivery," Curr. Drug targets 16(6), 571-581 (2015).

103. S. Kim, Y.-S. Chen, G. P. Luke, S. Y. Emelianov, "In vivo three-dimensional spectroscopic photoacoustic imaging for monitoring nanoparticle delivery," Biomed. Opt. Express 2(9), 2540-2550 (2011).

104. K. Homan, J. Shah, S. Gomez, H. Gensler, A. Karpiouk, L. Brannon-Peppas, S. Emelianov, "Silver nanosystems for photoacoustic imaging and image-guided therapy," J. Biomed. Opt. 15(2), 021316-021316-021319 (2010). 
105. C. M. Moore, D. Pendse, M. Emberton, "Photodynamic therapy for prostate cancer - A review of current status and future promise," Nat. Clin. Pract. Urol. 6(1), 18-30 (2009).

106. L. Xiang, D. Xing, H. Gu, D. Yang, S. Yang, L. Zeng, W. R. Chen, "Real-time optoacoustic monitoring of vascular damage during photodynamic therapy treatment of tumor," J. Biomed. Opt. $12(1), 014001$ (2007).

107. X. Huang, M. A. El-Sayed, "Gold nanoparticles: Optical properties and implementations in cancer diagnosis and photothermal therapy," J. Adv. Res. 1(1), 13-28 (2010).

108. J. Yuan, X. Yi, F. Yan, F. Wang, W. Qin, G. Wu, X. Yang, C. Shao, L. W. Chung, "Nearinfrared fluorescence imaging of prostate cancer using heptamethine carbocyanine dyes," Mol. Med. Rep. 11(2), 821-828 (2015).

109. R. Bouchard, O. Sahin, S. Emelianov, "Ultrasoundguided photoacoustic imaging: Current state and future development," IEEE Trans. Ultrason. Ferroelect. Freq. Control 61(3), 450-466 (2014). 